\title{
Algorithms for the assessment and management of insomnia in primary care
}

\author{
Donald Hilty' \\ Julie S Young' \\ James A Bourgeois' \\ Sally Klein ${ }^{2}$ \\ Kimberly A Hardin ${ }^{3}$ \\ 'Department of Psychiatry \\ and Behavioral Sciences; ${ }^{2}$ UC Davis \\ Medical Center; ${ }^{3}$ Sleep Medicine \\ Fellowship Training Program, \\ University of California Davis, \\ Sacramento, CA, USA
}

\begin{abstract}
Insomnia is a leading cause of sleep disturbance in primary care practice affecting $>30 \%$ of people in the United States and can result in psychological and physiological consequences. We aim for a focused discussion of some of the underpinnings of insomnia and practical tips for management (eg, algorithms). A PubMed search was conducted using English language papers between 1997-2007, with the terms "sleep," "insomnia"; "primary care" and "clinics"; "comorbid conditions"; "treatment" and "management." Sleep, psychiatric and medical disorders significantly affect sleep, causing patient suffering, potentially worsening of other disorders and increasing the use of primary care services. We provide an outline for practical assessment and treatment of insomnia in primary care, including the strengths and weaknesses of medications.
\end{abstract}

Keywords: insomnia, primary care, depression, anxiety, sleep, disorder

\section{Introduction}

Insomnia, defined as difficulty initiating and maintaining sleep, is the most common sleep disorder in the United States (US). ${ }^{1}$ About one-third of the adult population in the US experience insomnia at some point in their lives, ${ }^{2}$ and is a persistent problem in approximately $10 \%$ of US adults. ${ }^{3,4}$ The prevalence of insomnia increases with age, and is more common in women than in men. ${ }^{5}$ Lower socioeconomic status, divorce, widowhood, separation, recent life stressors, depression, and substance abuse increase the risk of insomnia. ${ }^{6,7}$ Insomnia has also been independently associated with worse health-related quality of life in outpatients at family medicine, internal medicine, endocrinology, cardiology, and psychiatry clinics. ${ }^{3,8,9}$ Women are twice as likely to suffer from insomnia than men, due to bodily changes (eg, pregnancy, menopause), or perhaps as some of the social factors (eg, unemployed or divorced) may be more common in women. ${ }^{4}$

Unfortunately, many primary care patients often have insomnia and may additionally complain of early awakening and nonrestorative sleep. ${ }^{2}$ This can result in numerous physical and psychological consequences, including medical (eg, blunted hypothalamic-pituitary-adrenal axis, and impaired host defenses) and psychiatric (eg, anxiety and mood, and cognitive) disorders. ${ }^{2}$

Normal sleep architecture refers to a characteristic pattern of sleep and consists of two major stages, nonrapid eye movement (NREM; comprises $75 \%-80 \%$ of total sleep) and rapid eye movement (REM). Normal adult sleep begins with NREM and alternates between NREM and REM every 90 minutes. Normal sleep latency is approximately 10-20 min and total sleep time (TST) averages 6-9 hours. NREM sleep is characterized by relatively quiescent brain activity, decreased metabolic rate, and slow-wave sleep, which are theorized to be necessary for physiologic restoration. Sleep is greatest when very young and least when old. 
The etiology of sleep disruption is multi-factorial and includes the patient's underlying illness(es), medical treatments, and biopsychosocial factors. The goals of this paper are: 1) to discuss factors that lead to insomnia in medical patients, 2) to provide a practical approach for working up insomnia, and 3) to offer principles and algorithms to guide management of insomnia.

\section{Factors affecting sleep}

Insomnia and other sleep disorders are caused by: medical, neurological, pain, and psychiatric disorders. In addition, medications and environmental factors can cause insomnia. Finally, conversely, sleep disorders can worsen medical conditions. This section provides an overview of these interactions.

\section{General medical disorders}

Causes of insomnia include sleep disordered breathing (SBD), congestive heart failure (CHF), diabetes mellitus, thyroid disorders, chronic obstructive pulmonary disease (COPD), gastroesophageal reflux (GER), cardiovascular disease, and renal disease. ${ }^{10}$

Problems such as frequent nocturnal awakenings in patients with congestive heart failure (CHF) may be related to orthopnea or paroxysmal nocturnal dyspnea, or may reflect undiagnosed SDB problems such as obstructive sleep apnea (OSA) or Cheyne-Stokes Breathing (CSB). Treatment of CSB with CPAP seems to improve sleep quality and ejection fraction in patients with CHF. ${ }^{11}$

Patients with COPD have decreased total sleep times (TST), SWS, and REM sleep. ${ }^{10}$ Shortness of breath, nocturnal cough, and wheezing worsen sleep. ${ }^{12}$ This leads to fatigue and sleep deprivation, which undermine the work of breathing and impair gas exchange. Airflow obstruction tends to worsen in the early morning hours in patients with COPD and asthma and may be related to REM effect on the diaphragm.

Approximately $50 \%$ of patients with chronic end-stage renal disease have insomnia and other sleep disorders. ${ }^{13}$ Patients complain of restless leg syndrome, periodic limb movement disorder, bone pain, nausea and pruritis. The etiology of sleep disorders appears to be related to metabolic derangements associated with renal disease or from co-existing diabetes.

\section{Endocrine disorders}

Endocrine disorders have also been associated with sleep disruption. Studies suggest that patients with diabetes mellitus (DM) have decreased TST and impaired sleep quality secondary ${ }^{14}$ to nocturia and neuropathic pain. ${ }^{15}$ There is accumulating evidence that poor sleep impairs glucose metabolic control. Inadequate quality or quantity of sleep is shown to be a risk factor for developing type 2 diabetes in large prospective studies, and predicted increased levels of HbAlc in type 2 diabetics. ${ }^{16}$

\section{Neurological disorders}

Since the brain and its various systems are critical in regulating sleep and wakefulness, patients with neurologic disorders have an increased risk of developing sleep disorders. Patients with dementia, other neurodegenerative disorders (eg, Parkinson's), epilepsy, and traumatic brain injury (TBI) have a higher prevalence of sleep disturbance and sleep disorders. ${ }^{17}$ Post-stroke patients can develop insomnia or hypersomnia, which is a reduction in sleep latency, increased sleep, or excessive daytime sleepiness. Brain-injured patients are also susceptible to sleep problems, with incidences as high as $72.7 \%$ in the hospital (mostly sleep initiation and maintenance) and $51.9 \%$ in the community (mostly excessive somnolence) $;{ }^{18}$ many with circadian rhythm sleep disorders (CRSD).

\section{Pain}

Between $50 \%$ and $70 \%$ of patients with chronic pain complain of impaired sleep. ${ }^{19}$ Sleep disruption is so common in fibromyalgia (75\%) that it is considered to be a key diagnostic symptom. ${ }^{20}$ In burn patients, pain was associated with increased intermittent awakenings, prolonged periods of wake time, poor pain tolerance, and complaints of higher pain intensity the following day. ${ }^{21}$ Pain increases cortical arousals and awakening, and sleep deprivation increases pain sensitivity and vulnerability to pain by inhibiting opioid protein synthesis and/or reducing opioid receptor affinity. ${ }^{21}$

\section{Women's health}

Women have a higher rate of insomnia than men, nearly 2:1 in one survey. ${ }^{4}$ Psychosocial risk factors may be higher in women and they also contest with significant changes during pregnancy. Peri- and post-menopausal changes (eg, nocturia, hot flashes) affect many women, ${ }^{22}$ though ageing associated with such change may account for differences compared to pre-menopausal women. ${ }^{23}$

\section{Psychiatric disorders}

Sleep problems are so common in psychiatric conditions that insomnia is listed as a symptom for mood (eg, manic or depressive), anxiety, and substance abuse disorders. 
Sleep disturbance in patients with undiagnosed psychiatric disorders may alert primary care providers to these problems. In a survey of 200 general medical patients in a hospital, $112(56.5 \%)$ complained of insomnia and 100 (50\%) met criteria for at least one psychiatric disorder - only $1.5 \%$ of those were charted - with depression most common at 35\%. ${ }^{24}$ The results showed that patients with insomnia had 3.6 times higher risk of having depression than those without insomnia.

An outpatient survey noted that chronic insomnia patients were 40 times more likely to have depression and six times more likely to have an anxiety disorder compared to those without insomnia. ${ }^{6}$ Longitudinal studies link prior insomnia with 2- to 5-times increased risk of mood and anxiety disorders and suicide. ${ }^{25}$

An estimated $65 \%$ with major depression have difficulty falling asleep, frequent awakenings, or early morning awakenings. ${ }^{26}$ Three patterns of sleep architecture abnormalities have been observed in patients with major depression: 1) sleep continuity disturbances characterized by prolonged sleep-onset, increased wake time during sleep, increased early morning wake time, and decreased total sleep time; 2) decreased proportion and length of SWS; and 3) REM sleep abnormalities such as reduced time to REM sleep, prolonged first REM sleep period, and increased REM sleep percentage. ${ }^{26}$

\section{Medications affecting sleep}

Sedatives and opioids may help with sleep onset, but actually impair sleep architecture (Table 1). Hypnotic agents with a significant anticholinergic component, such as diphenhydramine, are particularly prone to decrease cognitive performance and may precipitate frank delirium. Hypnotics are often prescribed and mainly work for initial insomnia, but can sometimes worsen sleep. Similarly, benzodiazepines can be problematic, particularly in older patients by causing motor impairment and increasing risk for delirium. Other common offenders are antihypertensives, antihistamines, corticosteroids, antiepileptics, beta antagonists (particularly lipophilic ones like propranolol and timolol) decrease REM sleep and increase nightmares and insomnia, anabolic steroids and bronchodilator therapy.

Medications used to treat common medical and psychiatric illnesses can also disrupt sleep (see Table 1). These include selective serotonin reuptake inhibitors (SSRIs), monoamine oxidase inhibitors (MAOIs), and tri- and tetracyclic agents (TCAs).

\section{Environmental factors}

Many hospitals have difficulty adhering to the World Health Organization's recommendation of keeping noise level to $\leq 35 \mathrm{~dB}$ in hospital rooms, particularly intensive care units (ICUs), daytime peaks up to $150-200 \mathrm{~dB}$ and nighttime peaks $>80 \mathrm{~dB}$. By comparison, a running vacuum cleaner is about $80 \mathrm{~dB}$. It follows, then, that residences, hotels and apartments have varying $\mathrm{dB}$ levels, often affecting sleep. Irregular sleep schedules, parenting young children and nighttime bathroom trips are common for many.

Circadian rhythm sleep disorders are disorders that are related to the timing of sleep within the 24-hour day. They include time-zone change syndrome (ie, jet lag), shift work sleep disorder, irregular sleep-wake pattern, delayed or advanced sleep phase syndromes, and non-24-hour sleep-wake disorder. Some of these disorders are influenced by an individual's control (eg, shift work or time zone change) or secondary to disorders of neurological mechanisms (eg, irregular sleep-wake pattern and advanced sleep phase syndrome).

\section{Affect of sleep disorders on medical disorders}

Data is mounting that primary sleep disorders, such as sleep disordered breathing (SDB), disturb sleep and exacerbate the pathophysiology associated with certain disease processes. Additionally, medical disease may directly impair sleep physiology leading to a cyclical interaction (eg, OSA leads to impaired sleep and increased heart rate and blood pressure, which then increases $\mathrm{CHF}$, which leads to impaired sleep). OSA is a common sleep disorder, and pertinent in this discussion as a chronic cause of sleep maintenance insomnia. It is characterized by complete or partial pharyngeal obstruction that leads to apnea, snoring, choking and daytime somnolence. ${ }^{27}$ SBD has been independently associated with cardiovascular diseases such as hypertension, heart failure, ischemic heart disease, atrial fibrillation, and cerebrovascular disease. ${ }^{28}$

\section{Assessment and evaluation of sleep History}

Analysis of sleep problems requires a focused, systematic assessment of potential factors that cause fragmented or interrupted sleep, as related by the patient and family. The history, physical examination and diagnostic tests yield important clues. Just as clinicians use pain as a clue to underlying illnesses, the practitioner should use insomnia to investigate for a cause, rather than immediately prescribing treatment (Figure 1).

First and foremost, gaining an initial sense of acute vs chronic complaints will help guide the nature, depth, and breadth of a focused interview about insomnia and its differential. Acute insomnia may last from a single night to 


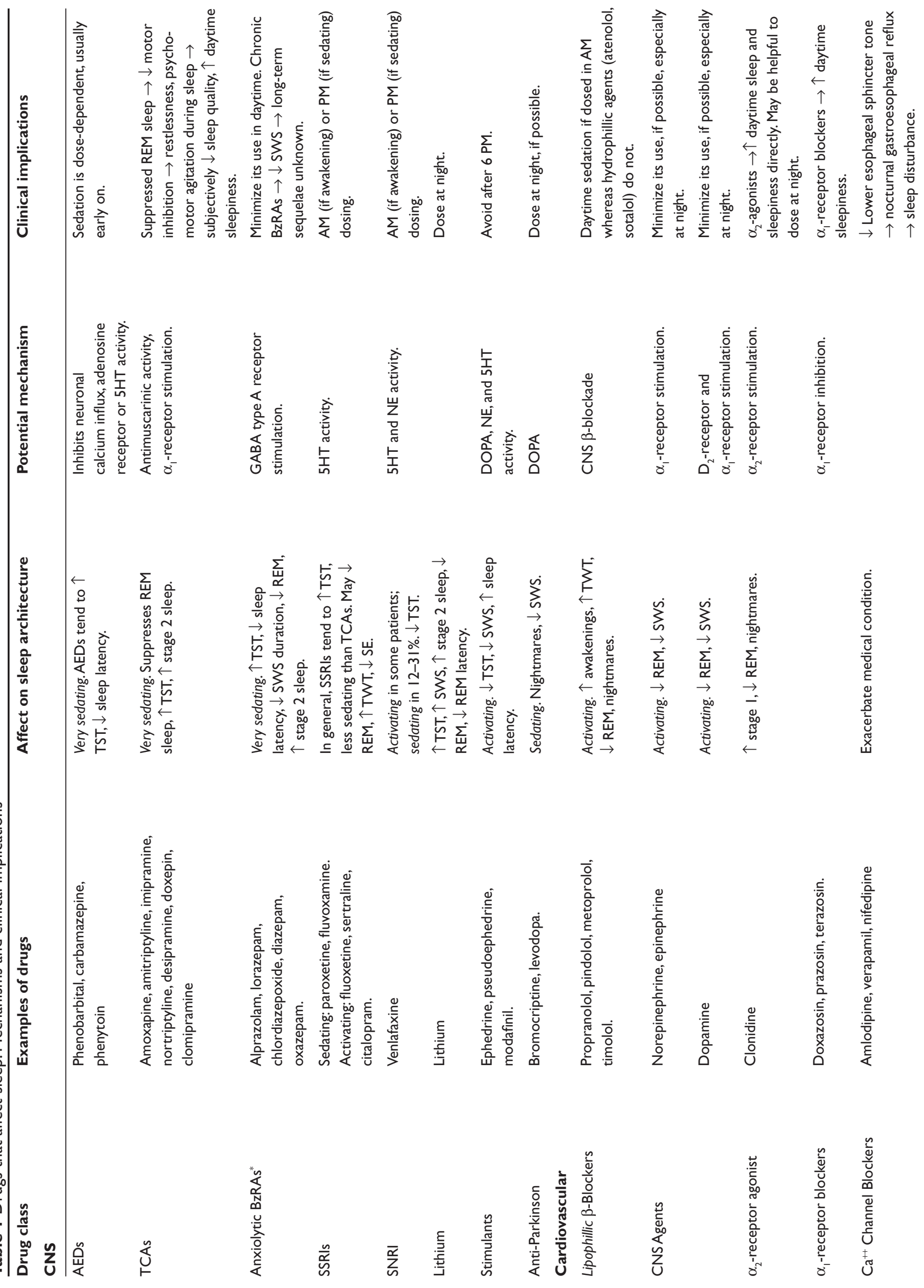



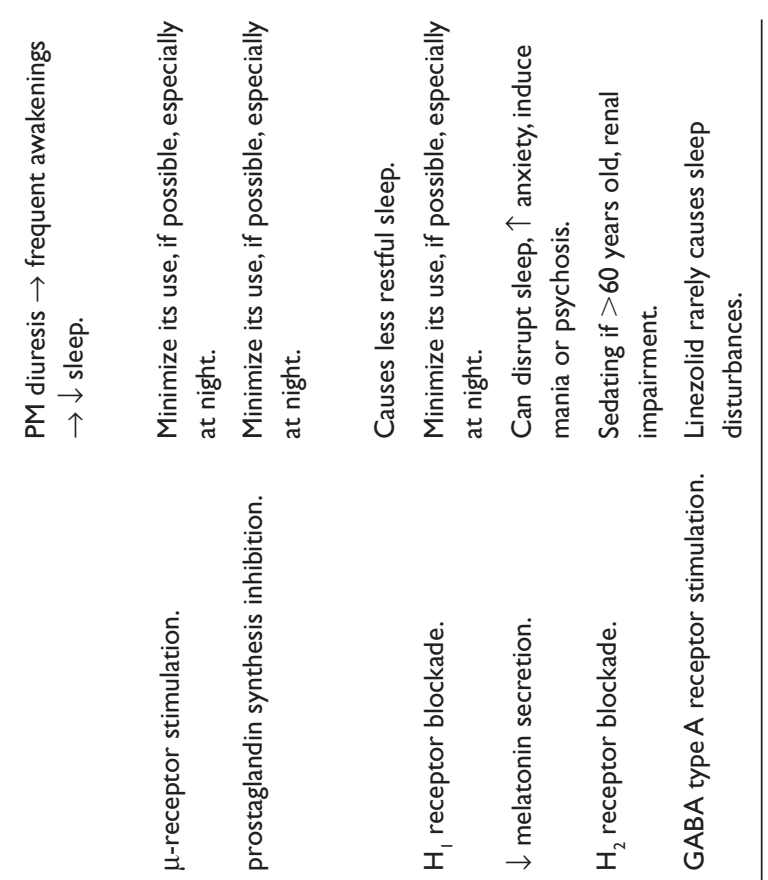

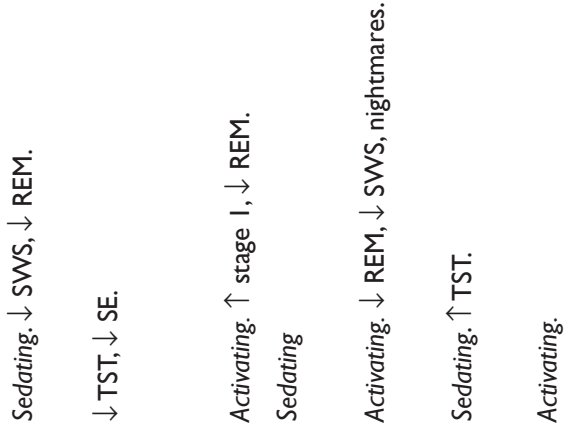

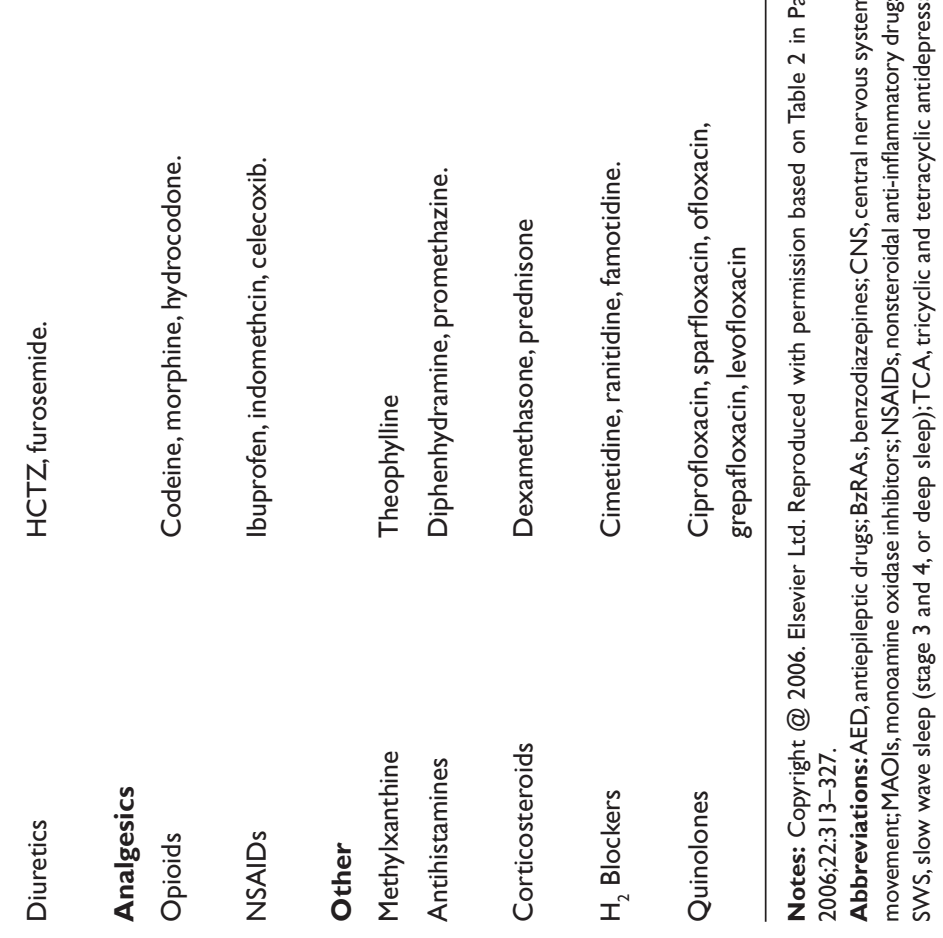




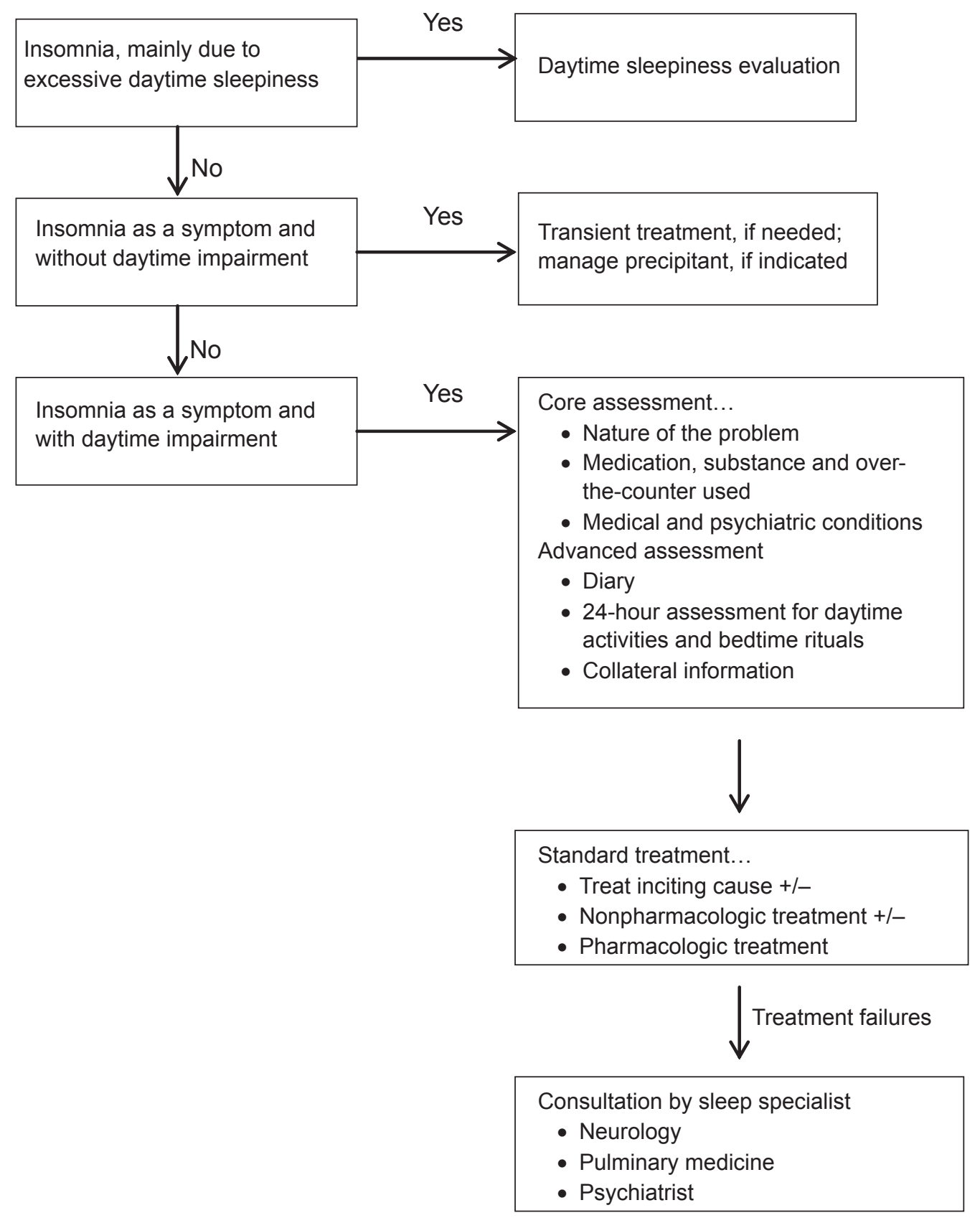

Figure I Overview of assessment and treatment of insomnia in primary care.

several weeks (stress, changes in schedule and time zone changes) may not require treatment. Mild insomnia often can be prevented or cured by practicing good sleep habits (see below). If the insomnia interferes with daily function because the patient is sleepy and tired, more investigation may be required, and sleeping pills may be indicated shortand long-term. Treatment for chronic insomnia includes first treating any underlying conditions or health problems that are causing the insomnia. Sleep habits, behavioral approaches and medications may be helpful.
Specific questions in the history to determine the exact nature of the complaint include:

- Do you have problems falling asleep or staying asleep?

- Do you frequently awaken, and is that associated with anything (eg, pain)?

- Do you awaken tired or unrefreshed despite many hours of sleep?

It is important to know the patient's usual sleep habits and behaviors - and for acute insomnia, recent changes - are associated with sleep hygiene. 
- What time do you go to bed and get up?

- Is the insomnia related to any new habits lately (eg, travelling) or adjustments in day-night changes (eg, new shift at work)?

- What activities do you normally have throughout the day?

- Have there been any recent changes in your routine or health?

- When going to sleep, how anxious are you or how mentally aroused are you?

The simplest method to ascertain the patient's routine is to have him/her describe a typical day. Patients' routines may be severely disrupted during illness or stress. Staying in bed for prolonged periods and sleeping during the day can impair nighttime sleep.

Chronic insomnia may require a personal or family history of over-the-counter medication, substance disorders, primary sleep disorders (eg, OSA, restless leg syndrome) or symptoms of common sleep problems.

- Do you snore when you sleep? If so, how often? Do family members complain that you snore very loudly? Does drinking alcohol before sleep worsen the snoring?

- Has anyone noticed that you appear to "stop breathing" while asleep?

- Have you recently increased or decreased the amount of alcohol you drink? By how much, and over what period of time?

- Do you use cocaine, methamphetamine, "speed," or other substances?

- Do you smoke? If so, when?

Patients may have underlying sleep and psychiatric disorders that they are unaware of, insufficiently diagnosed or simply forgotten when feeling ill. For example, OSA is often undiagnosed and may present with hypersomnlence or insomnia, exacerbated by illness. Snoring may be dismissed as insignificant or "normal" especially in male patients. Additional medical and psychiatric history should be obtained for anxiety, depression, substance and psychotic disorders. Alcohol and drug use can have profound effects on sleep, with the former normally suppressing REM sleep.

On more difficult patients, the provider can consider the following:

- 24-hour assessment of activities

- Diary logging daily activities and sleep patterns by the patient over 7-30 days

- Collateral information

\section{Physical examination}

Key issues on the physical examination include vital signs, signs of a medical condition (eg, obesity), changes from the last clinic visit, stigmata of substance use and mental status changes (mood, cognition, physical appearance).

\section{Diagnostic tests and procedures}

Primary care providers rarely order more advanced tests, instead deferring first to a consultation from neurology, pulmonology, psychiatry or other sleep specialists. Sleep is identified and quantified by polysomnography (PSG), but it is not that useful on most chronic insomnia patients. It may be useful with sleep apnea, periodic limb movements or parasomnias. Actigraphy is an objective means to assess rest activity patterns, by using a motion-sensitive bracelet on the non-dominant hand.

\section{Management strategy to improve sleep in primary care patients}

Optimizing sleep for patients includes nonpharmacological and pharmacological interventions. It is best not to assume that patients know or practice good sleep habits, or have regular sleep cycles. Those with a significant evidence base are noted with a * (some data) or ${ }^{* *}$ (significant and/or high quality data).

\section{Nonpharmacologic interventions}

- Reduce nocturnal discomfort

- Maximize pain management

- For patients with GER: No PO after 8PM and keep head of bed elevated

- Minimize iatrogenic insomnia

- Avoid benzodiazepines for sleep (unless withdrawal state)

- Avoid starting multiple medications and discontinue nonessential medications

- Change medication regimens to promote sleep (eg, give "activating" medications in the morning and sedating medications to the evening)

- Minimize opiates, antihistamines and anticholinergic agents

- Avoid night-time diuretics whenever possible

- Optimize nighttime glycemic control

- Promote good sleep hygiene

- No caffeine after 6 PM

- Appropriate lighting and same time to bed

- Encourage regular nocturnal sleep time, and discourage naps during the day

- Meditation, yoga and/or relaxation training as a ritual and/or to reduce tension and anxiety

- Exercise at least 3X/week before 6 PM 
- Improve the environment

- Provide adequate day and night lighting

- Offer sleep masks or ear plugs, if appropriate.

- Short- or long-term customized lifestyle planning

- May be pertinent to sleep-wake cycle disorders

- Targets emotional, cognitive and physical manifestations

- May be used with pharmacotherapy (eg, melatonin agents) (Renger 2008)

- Cognitive behavioral therapy (CBT)

A number of randomized clinical trials have been done with CBT, showing great promise in primary and secondary insomnias, with 1-4 sessions (often biweekly) having short- and long-term effect. ${ }^{29}$ Of interest is whether or not sedative hypnotics need to be discontinued for a CBT treatment, though in one study with clinical scales and most polysomnographic measures, CBT was highly effective with or without sedativehypnotic cessation. It produced the greatest changes in total sleep time, REM sleep and sleep efficiency. ${ }^{30}$ Finally, nurseadministered CBT has also been shown to be effective, which may have major implications for primary care practice. ${ }^{31}$

\section{Pharmacologic treatment}

Pharmacologic treatment, if necessary, complements the nonpharmacologic interventions. The clinician must:

- Decide on medications' likelihood of efficacy, side effects, and risks

- Find a fit between the patient, the type(s) of insomnia and the medication

- Consider Food and Drug Administration (FDA) indications (and contraindications), evidence base and off-label uses

- Choose a mechanism(s) of action in light of gammaaminobutyric acid, histamine, serotonin, melatonin, adenosine, corticotrophin-releasing factor and other systems being targeted

\section{Medication options}

(* = open or small sample data, vs** $=$ short-term, controlled data) vs*** $=$ short- and long-term

\section{controlled data)}

Medication options include the benzodiazepine $\mathrm{GABA}_{\mathbf{A}}$ receptor agonists $(\mathrm{BzRAs})^{* *}$, nonbenzodiazepine $\mathrm{GABA}_{\mathbf{A}}$ receptor agonists (non-BzRAs) ${ }^{* *}{ }^{* * *}$, melatonin-receptor agonists $^{* *}$, antidepressants (ADs) ${ }^{*}$, atypical antipsychotics (APs) ${ }^{*}$ and other medications* ${ }^{*}{ }^{32}$ The latter three groups are not FDA-approved for insomnia, but are commonly used. Suggestions for medication selection are based on the diagnosis, the evidence base and strength and weaknesses of the medications (Table 2 and Figure 2).

The FDA has approved three classes of medications for the treatment of insomnia: BzRAs, non-BzRAs and melatoninreceptor agonists. ${ }^{33}$ The number of comparison studies is limited, but the available data reveal that: 1) zolpidem (Ambien) may be better than temazepam (Restoril) in terms of sleep latency and quality; and 2) zaleplon (Sonata) may lead to a shorter sleep latency than zolpidem (Ambien), but the latter has longer sleep duration. ${ }^{34}$

BzRAs include estazolam, flurazepam, quazepam, temazepam, and triazolam. Though BzRAs decrease sleep latency and increase total sleep time, they also have adverse side effects such as anterograde amnesia, daytime sedation, cognitive impairment, poor motor coordination, dependence, tolerance, and rebound insomnia. ${ }^{6}$ Due to these side effects, BzRAs should be limited to generally healthy, young (eg, <45 years old) patients with initial insomnia and who are expected to have brief medication trials. BzRAs should be avoided in patients with a history of substance abuse, elderly patients, and others with a particularly high risk for falls or delirium (eg, traumatic brain injuries, strokes, multiple new medications).

Non-BzRAs seem to have a superior side effect profile when compared to BzRAs. Non-BzRAs include eszopiclone (Lunesta), zaleplon (Sonata), zolpidem (Ambien), and zolpidem extended-release. They have less next-day sedation, psychomotor dysfunction, or tolerance/withdrawal; less REM rebound; and lower abuse potential than BzRAs. ${ }^{34}$ Studies have included short-term effects for insomnia, ${ }^{34-36}$ short-term effect in comorbid medical and psychiatric disorders (eszopiclone) $)^{37 *}$ and long-term effects (eszopiclone; ${ }^{38}$ zolpidem extendedrelease $\left.^{39}\right)^{* * *}$. The sole melatonin-receptor agonist, ramelteon (Rozerem), also reduces time to fall asleep without next-day psychomotor and memory effects* ${ }^{*}{ }^{40}$ Ramelteon is believed to target receptors melatonin 1 and 2 receptors located in the brain's suprachiasmatic nucleus to stabilize circadian rhythms and stabilize the sleep-wake cycle. ${ }^{41}$

Limited data exist on the efficacy of non-FDA approved medications such as ADs and APs for insomnia. ${ }^{42}$ Sedating antidepressants that may be useful for some hospitalized patients, include trazodone (Desyrel) and mirtazapine (Remeron). Trazodone and mirtazepine are used most noticeably, though the former is associated with priapism in approximately 1:10,000 males. They may be an acceptable short-term alternative to BzRAs, but data are limited on them and recommended duration of use is unclear. Short-term 


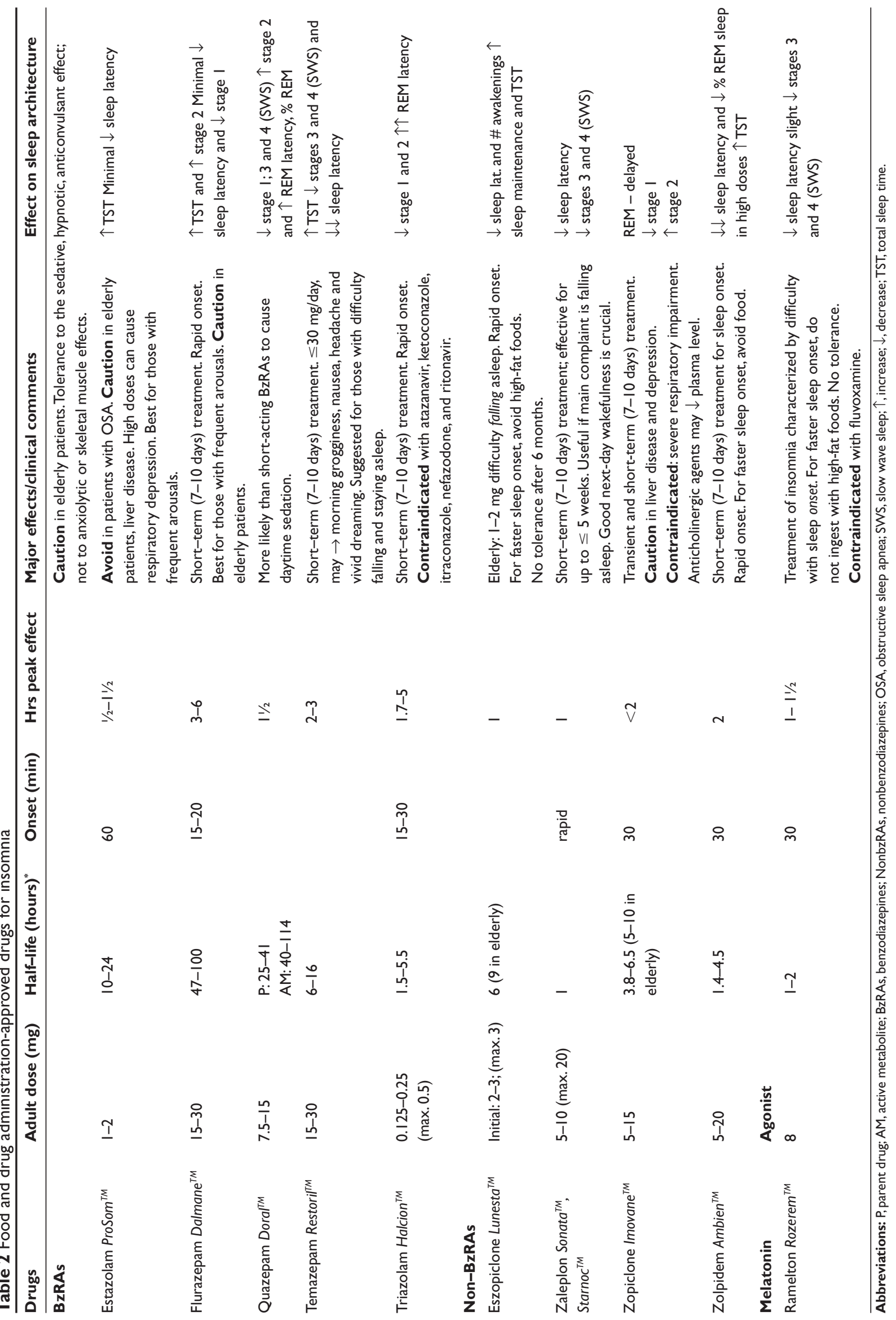




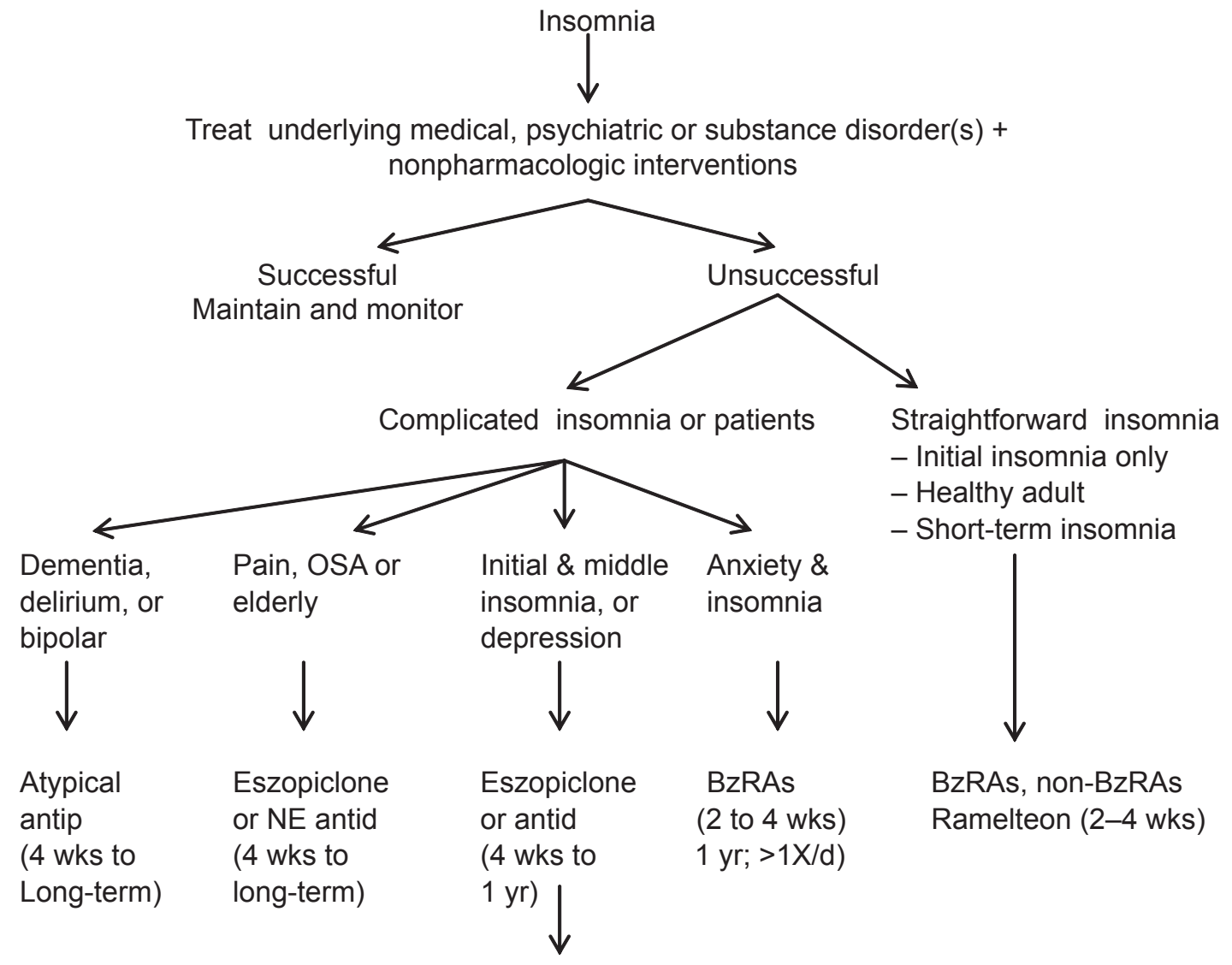

Failed trial, side effects or worsening insomnia 1

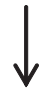

Try other agent(s) or classes

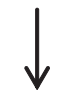

Failed trial, side effects or worsening insomnia 2<smiles>C=[V]</smiles>

Consultation with sleep specialist

Figure 2 Pharmacologic treatment algorithm for insomnia in primary care.

Note: Duration based on clinical experience and evidence base; widely variable in practice; for I yr + , re-evaluate often.

Abbreviations: BzRAs, benzodiazepines; Non-BzRAs, nonbenzodiazepines; OSA, obstructive sleep apnea.

studies have shown that low-dose trazodone $(50 \mathrm{mg})$ is less efficacious than zolpidem. Mirtazapine, which promotes both sleep and appetite, may be particularly helpful for patients with cancer, AIDS and other conditions in which the trio of poor sleep, anorexia, and depression are common. Although sedating, tricyclic antidepressants (TCAs) are not recommended to such patients because they increase the risk of cardiac conduction abnormalities, reduce the seizure threshold, and have significant anticholinergic effects.
Antipsychotics are not routinely used first-line for the treatment of insomnia, except in patients who are in the midst of acute manic or psychotic episodes. ${ }^{43}$ They typically increase sleep continuity and do not affect sleep architecture much. Sedating APs include risperidone (Risperdal; 0.5-2 mg HS), olanzapine (Zyprexa; 2.5-10 mg HS), quetiapine (Seroquel 12.5-300 mg HS); ziprasidone (Geodon) and aripiprazole (Abilify) are not usually sedating. Delirious patients or elderly patients with dementia who have 
nightly episodes of confusion, agitation, and cognitive decline ("sun-downing") may benefit from a low dose of a sedating AP to promote sleep and to prevent or treat these episodes. Common side effects include sedation, weight gain and metabolic changes (glucose, cholesterol, lipids). Rare, but important side effects, include neuroleptic malignant syndrome (a triad of mental status changes, increased muscle tone and autonomic hyperactivity), tardive dyskinesia, and akithisia (restlessness or inability to sit still).

The administration of antihistamines, barbiturates, chloral hydrate, and alternative/herbal therapies has been discouraged in recent years, since the benefits rarely outweigh the risks associated with their use. In the US, antihistamines such as diphenhydramine are the most commonly used over the counter agent for chronic insomnia. ${ }^{2}$ However, anticholinergic action of antihistamines may lead to orthostatic hypotension, and induce delirium in vulnerable patients, especially the elderly, and should be avoided if possible.

\section{Conclusion}

Sleep, medical and psychiatric disorders significantly affect sleep in primary care patients, as do some medical treatments. More research is needed to evaluate if/how screening, education, and good habits help patients, with or without medication. Medications have strengths and weaknesses that need to be considered in the process of selection. Algorithms for diagnosis and management may serve as practical guides for primary care providers. More research is needed on the treatment of insomnia in primary care practice.

\section{Disclosure}

The authors report no conflicts of interest in this work.

\section{References}

1. International Classification of Sleep Disorders. American Academy of Sleep Medicine, One Westbrook Corporate Center, Westchester, IL, 60154, 2001

2. National Institute of Health. NIH state-of-the-science conference statement on manifestations and management of chronic insomnia in adults. NIH Consens Stat Sci Statements. 2005;22:1-30.

3. Katz DA, McHorney CA. The relationship between insomnia and health-related quality of life in patients with chronic illness. J Fam Pract. 2002;51:229-235.

4. National Sleep Foundation. 2005. Cited Oct 6, 2008. Available from: http://www.sleepfoundation.org.

5. Bliwise DL. Review: Sleep in normal aging and dementia. Sleep. 1993;16:40-81.

6. Ford DE, Kamerow DB. Epidmiologic study of sleep disturbances and psychiatric disorders: An opportunity for prevention? JAMA. 1989;262:1479-1484.

7. Zorick FJ. Evaluation and management of insomnia: An overview. In: Kryger MH, Roth T, Dement WC (editors). Principles and Practice of Sleep Medicine. 3rd Ed. Philadephia: W.B. Saunders Company; 2000. p. 615-616.
8. Richardson G. Update on the science, diagnosis and management of insomnia. London: Royal Society of Medicine Press Ltd, International Congress and Symposium Series; 2006. p. 262.

9. BaHamman A. Sleep in acute care units. Sleep Breath. 2006;10:6-15.

10. Ballard RD. Sleep and medical disorders. Prim Care Clin Office Pract. 2005;35:511-533.

11. Berry RB, Harding SM. Sleep and medical disorders. Med Clin N Am. 2004;88:679-703.

12. Mohsenin V. Sleep in chronic obstructive pulmonary disease. Sleep Med Clin. 2007;2:1-8.

13. Hanly P. Sleep disorders and end-stage renal disease. Sleep Med Clin. 2007;2:59-66.

14. Gislason T, Almqvist M. Somatic diseases and sleep complaints: An epidemiologic study of 3,201 Swedish men. Acta Med Scand. 1987;221:475-481.

15. Lamond N, Tiggermann M, Dawson D. Factors predicting sleep disruption in type II diabetes. Sleep. 2000;23:415-416.

16. Yaggi HK, Araujo AB, McKinlay JB. Sleep duration as a risk factor for the development of type 2 diabetes. Diabetes Care. 2006;29:657-661.

17. Hoyt BD. Sleep in patients with neurologic and psychiatric disorders Prim Care Clin Off Pract. 2005;32:535-548.

18. Fichtenberg NL, Zafonte RD, Putnam S, Mann NR, Millard AE. Insomnia in a post-acute brain injury sample. Brain Inj. 2002;16:197-206.

19. Barczi SR, Juergens TM. 2006. Comorbidities: Psychiatric, medical, medications, and substances. Sleep Med Clin. 2006;1:231-245.

20. Lineberger MD, Means JK, Edinger JD. Sleep disturbance and fibromyalgia. Sleep Med Clin. 2007;2:31-39.

21. Raymond I, Ancoli-Israel S, Choiniere M. Sleep disturbances, pain and analgesia in adults hospitalized for burn injuries. Sleep Med. 2004;5:551-559.

22. United States Health and Human Services. Frequently asked questions. 2008. Cited Oct 6, 2008. Available from: http:/www.4woman.gov/ FAQ/insomnia.htm.

23. Kalleinen N, Polo-Kantola P, Himanen SL, et al. Sleep and the menopause - do postmenopausal women experience worse sleep than premenopausal women? Menopause Int. 2008;14:97-104.

24. Rocha FL, Hara C, Rodriguez CV, et al. Is insomnia a marker for psychiatric disorders in general hospitals? Sleep Med. 2005;6:549-553.

25. Weissman MM, Greenwald S, Nino-Murcia G, et al. The morbidity of insomnia uncomplicated by psychiatric disorders. Gen Hosp Psych. 1997; 19:245-250.

26. Peterson MJ, Benca RM. Sleep in mood disorders. Psychiatr Clin N Am. 2006;29:1009-1032.

27. Bassiri AG, Guilleminault C. Clinical features and evaluation of obstructive sleep apnea-hypopnea syndrome. In: Kryger MH, Roth T, Dement WC (editors). Principles and Practice of Sleep Medicine. 3rd Ed. Philadephia: W.B. Saunders Company; 2000. p. 869-872.

28. Wattanakit E, Boland L, Punjabi NM, et al. Relation of sleep-disordered breathing to carotid plaque and intima-media thickness. Atherosclerosis. In press.

29. Edinger JD, Wohlgemuth WK, Radtke RA, et al. Dose-response effects of cognitive-behavioral insomnia therapy: a randomized clinical trial. Sleep. 2007;30:203-212.

30. Zavesicka L, Brunovsky M, Matousek M, et al. Discontinuation of hypnotics during cognitive behavioural therapy for insomnia. $B M C$ Psychiatry. 2008;8:80

31. Espie CA, MacMahon KM, Kelly HL, et al. Randomized clinical effectiveness trial of nurse-administered small-group cognitive behavior therapy for persistent insomnia in general practice. Sleep. 2007;30:574-584.

32. Green BT, Broughton WA, O’Connor JB. Marked improvement in nocturnal gastroesophageal reflux in a large cohort of patients with obstructive sleep apnea. Arch Intern Med. 2003;163:41-45.

33. Neubauer DB. State-of-the-art sleep management. Awakening insomnia management. Proceedings from a satellite symposium at SLEEP 2006: 20th Anniversary Meeting of the Associated Professional Sleep Societies, Salt Lake City, UT, June 20. 2006, p. 6-12. 
34. Dundar Y, Boland A, Strobl J, et al. Newer hypnotic drugs for the short-term management of insomnia: A systematic review and economic evaluation. Health Tech Ass. 2004;19:305-322.

35. Pagel JF. Medications and their effect on sleep. Prim Care Clin Off Pract. 2005;32:401-509.

36. Erman MK, Zammit G, Rubens R, et al. A polysomnographic placebo-controlled evaluation of the efficacy and safety of eszopiclone relative to placebo and zolpidem in the treatment of primary insomnia. J Clin Sleep Med. 2008;4:229-234.

37. Pollack M, Kinrys G, Krystal A, et al. Eszopiclone coadministered with escitalopram in patients with insomnia and comorbid generalized anxiety disorder. Arch Gen Psychiatry. 2008;65:551-562.

38. Walsh JK, Krystal AD, Amato DA, et al. Nightly treatment of primary insomnia with eszopiclone for six months: effect on sleep, quality of life, and work limitations. Sleep. 2007;30:959-968.
39. Krystal AD, Erman M, Zammit GK, et al. Long-term efficacy and safety of zolpidem extended-release $12.5 \mathrm{mg}$, administered 3 to 7 nights per week for 24 weeks, in patients with chronic primary insomnia: a 6-month, randomized, double-blind, placebo-controlled, parallel-group, multicenter study. Sleep. 2008;31:79-90.

40. Erman M, Seiden D, Zammit G, et al. An efficacy, safety, and dose-response study of Ramelteon in patients with chronic primary insomnia. Sleep Med. 2006;7:17-24.

41. Turek FW, Gillette MU. Melatonin, sleep, and circadian rhythms: Rationale for development of specific melatonin agonists. Sleep Med. 2004;5:523-532.

42. Infante M, Benca R. Treatment of insomnia. Primary Psychiatry. 2005; 12:47-56.

43. Hilty DM, Leamon ML, Lim RF, et al. Diagnosis and treatment of bipolar disorder in the primary care setting. Primary Psychiatry. 2006;13:77-85. 